\title{
Prácticas de alimentación, actividad física y condición física de niños preescolares españoles. Influencia de variables sociodemográficas
} Feeding practices, physical activity, and fitness in Spanish
preschoolers. Influence of sociodemographic outcome measures

Dr. Pedro Á. Latorre Román ${ }^{a}$, Lic. David Mora López y Lic. Felipe García Pinillos ${ }^{a}$

\section{RESUMEN}

Introducción. La edad preescolar es un período esencial para establecer hábitos de nutrición y actividad física adecuados.

Objetivo. Elpropósito deesteestudio fue analizar el estado nutricional, el nivel de actividad física (AF) y la condición física (CF) de niños preescolares en relación con el sexo y con las variables sociodemográficas de los padres.

Material y métodos. Se incluyeron niños preescolares seleccionados de 30 centrosescolares del sur de España. Se registraron parámetros de $\mathrm{CF}, \mathrm{AF}$, antropometría, estado nutricional de los niños y variables sociodemográficas de los padres.

Resultados. Participaron 1287 niños de entre 3 y 6 años, 643 niños y 644 niñas, y 1267 padres (el $72,4 \%$ eran madres y el $27,6 \%$, padres). Las niñas presentaron niveles más bajos de sobrepeso y obesidad que los niños. Existieron diferencias significativas por sexos en el consumo de determinados alimentos: mayor consumo de lácteos en el desayuno y aceite de oliva en niñas y mayor consumo de comidas rápidas y pastas o arroz en los varones. Los varones presentaron una mejor CF. Los niños del estrato socioeconómico más bajo mostraron mayor índice de masa corporal, peor estado nutricional y más bajo nivel de AF. Los niños de padres con estudios universitarios presentaron menoríndice de masa corporal y mejor estado nutricional.

Conclusiones. Los niños preescolares de este estudio presentaron valores elevados de sobrepesoy obesidad y bajo nivel de $\mathrm{AF}$, teniendo en cuenta las referencias internacionales. Las niñas mostraron una $\mathrm{CF}$ inferior a la de los varones. Los niños cuyos padres presentaron un nivel socioeconómico bajo y sin estudios mostraron un nivel nutricional precario.

Palabras clave: preescolar, aptitud física, actividad física, obesidad, nutrición.

http:/ /dx.doi.org/10.5546/aap.2016.441

\section{INTRODUCCIÓN}

El incremento del sobrepeso y la obesidad en el mundo entero es una realidad y es uno de los mayores retos para la protección de la salud.
La prevalencia mundial de sobrepeso y obesidad infantil aumentó del $4,2 \%$ en 1990 al $6,7 \%$ en 2010 . Con esta tendencia, se espera llegar al $9,1 \%$ en 2020; todo ello como consecuencia de un probable cambio en los patrones de nutrición y de actividad física (AF). ${ }^{1}$ Además, la adiposidad, la baja condición física (CF) aeróbica y los bajos niveles de AF están asociados con el riesgo de enfermedad cardiovascular en los niños y su alta prevalencia representa un importante problema de salud pública. ${ }^{2}$

Es necesario analizar determinados factores modificables (influencia de los padres, entorno social, colegios, tiempo de ocio, etc.) y no modificables (sexo y edad) para poder realizar un abordaje integral del sobrepeso y la obesidad en niños preescolares y su relación con la AF. Dentro de los factores modificables, se destaca la $\mathrm{AF}$, la cual se asocia de manera inversa con el sobrepeso. ${ }^{3}$ Varios estudios han demostrado que la AF de los niños en edad preescolar es moderadamente baja..$^{4-6}$ Además, la CF es un biomarcador importante de la salud desde una edad temprana. ${ }^{7}$ El sobrepeso y la adiposidad influyen negativamente en la CF de los niños, y existe una importante conexión entre el crecimiento del cuerpo y la CF.9,10 Además del ejercicio físico, la alimentación es otro de los factores exógenos que más importancia tienen en el crecimiento infantil.

La edad preescolar es un momento idóneo para establecer hábitos de nutrición y AF adecuados; es un período crítico para la prevención de 
la obesidad, la cual se asocia con consecuencias para la salud que pueden persistir en la adolescencia y la edad adulta. , $^{8,112}$ Por tanto, la evaluación del estado nutricional y el nivel de $\mathrm{AF}$ en niños preescolares debe ser un elemento esencial para el control y el seguimiento de la salud de esta población.

Teniendo en cuenta la información presentada anteriormente, se plantea como hipótesis que es posible que, a edades preescolares, las prácticas alimenticias y la AF puedan verse afectadas por el sexo de los niños y por determinadas características sociodemográficas de los padres. El objetivo de este estudio fue evaluar el estado nutricional, ponderal, el nivel de AF y CF de niños preescolares en relación con el sexo y otras variables sociodemográficas de los padres.

\section{POBLACIÓN Y MÉTODOS}

Se incluyeron niños preescolares pertenecientes a 30 centros escolares del sur de España seleccionados aleatoriamente. Como criterios de inclusión, se establecieron la escolarización en Educación Infantil y no padecer discapacidad física y/o intelectual. Los padres firmaron un consentimiento informado de participación voluntaria en esta investigación (determinante para la inclusión de los niños). El estudio se completó de acuerdo con las normas de la Declaración de Helsinki (versión 2013). El estudio fue aprobado por el Comité de Ética de la Universidad de Jaén.

Mediante un cuestionario sociodemográfico realizado ad hoc, se recogió información de los padres, como nivel de estudios, estado civil y nivel socioeconómico (sobre la base de su autopercepción socioeconómica). Como parámetros antropométricos, se analizaron la altura $(\mathrm{cm})$, que se midió con un estadiómetro (Seca 222, Hamburgo, Alemania), el peso (kg), que se midió con una báscula (Seca 634, Hamburgo, Alemania), y el índice de masa corporal (IMC), que se obtuvo de la ecuación IMC= peso $(\mathrm{kg}) /$ talla $(\mathrm{m})^{2}$. Además, se registró el contorno del abdomen a nivel de la cicatriz umbilical mediante una cinta ergonómica Seca 201. En el análisis de la CF, se empleó la batería de pruebas de Latorre et al. (2015) ${ }^{13}$ que representa componentes básicos de la condición físico-motriz, como la resistencia, la fuerza, la velocidad y el equilibrio. Además, se añadió la prueba de dinamometría manual para analizar la fuerza de prensión manual. El análisis del estado nutricional, tiempo de uso de pantallas (se refiere al uso de pantallas de video, como televisión, tabletas, consolas, móviles, computadoras, etc.) y AF se realizó mediante el cuestionario Krece Plus, el cual presenta 16 cuestiones referentes a la ingesta nutricional y dos cuestiones sobre uso de pantallas y AF extraescolar. ${ }^{14}$ El registro del tiempo en sedestación de los padres se realizó mediante el análisis del ítem 7 del International Physical Activity Questionnaire (IPAQ) $)^{15}$ en su versión corta.

Todas las pruebas se realizaron en los centros escolares, en sus instalaciones deportivas y aulas, por investigadores expertos y en presencia de los docentes de cada grupo de alumnos. Los padres cumplimentaron, de manera autoadministrada, los diferentes cuestionarios en su hogar. Posteriormente, en dos sesiones separadas por 48 horas, los niños fueron evaluados en la jornada escolar (de 9 a 14), con una duración aproximada de 40 minutos por sesión. En el primer día, se realizaron las pruebas de fuerza de prensión manual (dos intentos con cada mano), equilibrio (dos intentos con cada pierna) y salto horizontal (dos intentos). El segundo día, se registraron la prueba de $20 \mathrm{~m}$ (dos intentos) y 10 × $20 \mathrm{~m}$ (un intento). Previamente a la realización de las pruebas físicas, los niños efectuaron un calentamiento basado en carrera continua y movilidad articular; además, se realizaron, por parte del equipo investigador, pruebas de demostración y los niños ejecutaron ensayos de familiarización. Se seleccionaron los mejores intentos en cada prueba, excepto en la dinamometría manual y equilibrio, que se realizó un promedio de las dos manos y piernas, respectivamente, con el mejor intento. Todos los niños fueron motivados para desarrollar el máximo rendimiento físico mediante instrucciones verbales que los animaran a correr más rápido, saltar más lejos, etc.

El tamaño de la muestra para una población infinita con prevalencia desconocida en la que $p=q=0,50$, con un nivel de confianza del 99\% y un error del $5 \%$ fue de 645 sujetos. Para la selección de la muestra, se emplearon los datos de la Junta de Andalucía sobre Centros de Educación Infantil de Andalucía.

Los datos de este estudio se analizaron mediante el programa estadístico SPSS, v.19.0 para Windows (SPSS Inc., Chicago, USA). El nivel de significación se fijó en $p<0,05$, a un nivel de confianza del 95\%. Los datos se mostraron en estadísticos descriptivos de media, desviación típica y porcentajes. Se comprobó la distribución normal de los datos y la homogeneidad de varianzas mediante pruebas de Kolmogorov- 
Smirnov y contraste de Levene, respectivamente. Las diferencias entre sexos, nivel de estudios y estado socioeconómico de los padres se analizaron mediante el análisis de varianza (analysis of variance; ANOVA, por sus siglas en inglés) con prueba post hoc mediante el ajuste de Bonferroni. El análisis del estado ponderal y del cuestionario Krece Plus por ítems en relación con el sexo se realizó mediante la prueba chi cuadrado. Por último, se realizó la correlación de Pearson entre las diferentes variables.

TABla 1. Características sociodemográficas de los padres

\begin{tabular}{lc}
\hline Estado civil & $\mathbf{n}(\%)$ \\
\hline Soltero & $53(4,2)$ \\
Casado o en pareja & $826(65,2)$ \\
Divorciado/separado & $372(29,4)$ \\
$\quad$ Viudo & $16(1,2)$ \\
Total & $1267(100)$ \\
Nivel socioeconómico & \\
$\quad$ Bajo & $107(8,6)$ \\
$\quad$ Medio & $814(65,6)$ \\
$\quad$ Alto & $319(25,8)$ \\
$\quad$ Total & $1240(100)$ \\
Nivel de estudios & \\
$\quad$ Sin estudios & $32(2,5)$ \\
$\quad$ Primarios & $316(25)$ \\
Secundarios & $483(38,2)$ \\
Universitarios & $433(34,3)$ \\
Total & $1264(100)$ \\
Tiempo de sedestación diario en minutos & \\
Media (DT) & $248,20(168,31)$ \\
\hline
\end{tabular}

DT: desviación típica.

\section{RESULTADOS}

El estudio se realizó entre los meses de abril y mayo de 2015. Participaron un total de 1287 niños de entre 3 y 6 años (edad $=50,90$ meses; $\left.\mathrm{IMC}=16,03 \pm 2,13 \mathrm{~kg} / \mathrm{m}^{2}\right), 643$ niños y 644 niñas, y 1267 padres (el 72,4\% eran madres y el $27,6 \%$, padres).

En la Figura 1, se expone el diagrama de flujo de los participantes. En la Tabla 1, se presentan las variables sociodemográficas en relación con uno de los padres.

En cuanto al estado ponderal por sexos, las diferencias fueron significativas $(p=0,004)$; las niñas presentaron niveles más bajos de sobrepeso y obesidad que los niños, $7,0 \%-9,2 \%$ vs. 9,7\%$13,9 \%$, respectivamente, y la prevalencia total de sobrepeso y obesidad fue de $8,3 \%$ y $11,5 \%$, respectivamente.

FIGURA 1. Diagrama de flujo de los participantes

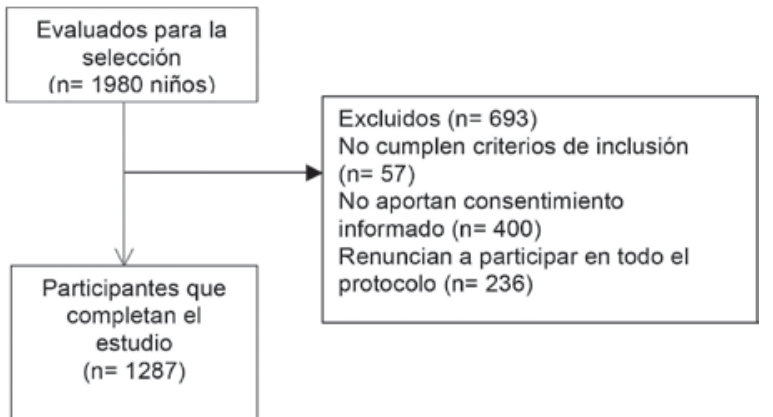

Tabla 2. Resultados del cuestionario Krece Plus en porcentaje de respuesta de los padres por cada ítem en la muestra total y por sexos

\begin{tabular}{|c|c|c|c|c|c|}
\hline Ítems & $\begin{array}{c}\mathbf{n} \\
\text { padres }\end{array}$ & $\begin{array}{c}\text { Porcentaje } \\
\text { total }\end{array}$ & $\begin{array}{c}\text { Niña } \\
(\%)\end{array}$ & $\begin{array}{c}\text { Niño } \\
(\%)\end{array}$ & $p$-valor \\
\hline No desayuna. & 1248 & 1,5 & 1,5 & 1,6 & 0,817 \\
\hline Desayuna un lácteo (leche o derivados). & 1257 & 93,3 & 94,9 & 91,7 & 0,024 \\
\hline Desayuna un cereal o derivado. & 1254 & 68 & 66,6 & 69,4 & 0,290 \\
\hline Desayuna bollos o dulces. & 1243 & 27,8 & 26,9 & 28,8 & 0,442 \\
\hline Ingiere una fruta o jugo de fruta todos los días. & 1263 & 79,2 & 78,4 & 79,9 & 0,516 \\
\hline Ingiere una segunda fruta todos los días. & 1257 & 38,9 & 37,7 & 40,2 & 0,360 \\
\hline Ingiere un segundo lácteo durante el día. & 1257 & 85,7 & 85,6 & 85,9 & 0,862 \\
\hline Ingiere regularmente verduras frescas o cocinadas una vez al día. & 1265 & 62,9 & 63,9 & 61,8 & 0,439 \\
\hline Ingiere verduras frescas o cocinadas más de una vez al día. & 1262 & 23 & 21,8 & 24,2 & 0,315 \\
\hline Ingiere pescado regularmente (más de 2 o 3 veces por semana). & 1264 & 76,9 & 77,5 & 76,3 & 0,617 \\
\hline Come una o más veces a la semana hamburguesas, panchos (hot dogs) o pizza. & 1266 & 29,7 & 25 & 34,3 & $<0,001$ \\
\hline Le gustan las legumbres (lentejas, judías, más de una vez a la semana). & 1267 & 84,1 & 84,7 & 83,5 & 0,572 \\
\hline Ingiere varias veces al día dulces y golosinas. & 1266 & 22,9 & 23 & 22,8 & 0,930 \\
\hline Ingiere pasta o arroz casi a diario (más de 5 veces por semana). & 1267 & 31,6 & 28,3 & 34,9 & 0,012 \\
\hline Utiliza aceite de oliva en su casa. & 1267 & 95,6 & 97,1 & 94,1 & 0,008 \\
\hline Toma bebidas alcohólicas ( $\geq 1$ /semana). & 1266 & 0 & 0 & 0 & -- \\
\hline
\end{tabular}


En la Tabla 2, se muestran los resultados del cuestionario Krece Plus en porcentaje de respuesta por cada ítem. Existieron diferencias significativas por sexos en el consumo de determinados alimentos: mayor consumo de lácteos en el desayuno y aceite de oliva en las niñas y mayor consumo de comidas rápidas y pastas o arroz en los varones.

En la Tabla 3, se exponen los resultados de las diferentes variables teniendo en cuenta el sexo. Se encontraron diferencias significativas en el IMC, que fue mayor en los niños. A su vez, estos manifestaron mejor rendimiento en salto horizontal, velocidad, resistencia y fuerza de prensión manual.
En la Tabla 4, se presentan los resultados de las diferentes variables teniendo en cuenta el nivel socioeconómico de los padres. Los niños del estrato socioeconómico más bajo mostraron mayor IMC, peor estado nutricional y más bajo nivel de AF. El tiempo de uso de pantallas fue menor en el estrato socioeconómico más elevado, que, sin embargo, presentó menor rendimiento en pruebas físicas, como el salto horizontal y la velocidad.

En la Tabla 5, se exponen los resultados de las diferentes variables teniendo en cuenta el nivel de estudios de los padres. Los niños de padres con estudios universitarios presentaron menor IMC, mejor estado nutricional y mayor salto horizontal.

TABLA 3. Edad, variables antropométricas, estado nutricional, nivel de actividad física, tiempo de uso de pantallas y condición física por sexos

\begin{tabular}{lccc}
\hline & $\begin{array}{c}\text { Niño } \\
\text { Media (DT) } \\
\mathbf{n}=\mathbf{6 4 3}\end{array}$ & $\begin{array}{c}\text { Niña } \\
\text { Media (DT) } \\
\mathbf{n = 6 4 4}\end{array}$ & $p$-valor \\
\hline Edad (meses) & $51,13(10,41)$ & $50,68(10,86)$ & 0,493 \\
IMC (kg/m $)$ & $16,19(2,17)$ & $15,87(2,08)$ & 0,007 \\
Circunferencia de cadera (cm) & $55,01(5,71)$ & $55,46(5,92)$ & 0,228 \\
Puntuación total Krece Plus (0-10) & $6,52(2,06)$ & $6,56(2,00)$ & 0,716 \\
Actividad física semanal (horas) & $1,87(1,63)$ & $1,71(1,55)$ & 0,079 \\
Tiempo de uso de pantallas diario (horas) & $3,14(6,64)$ & $3,13(6,63)$ & 0,971 \\
Salto horizontal (cm) & $73,28(25,83)$ & $65,98(25,55)$ & $<0,001$ \\
Velocidad, 20 m (s) & $6,16(1,21)$ & $6,55(1,26)$ & $<0,001$ \\
Resistencia (s) & $83,04(19,54)$ & $85,75(19,65)$ & 0,016 \\
Equilibrio (s) & $8,85(9,67)$ & $9,30(10,43)$ & 0,435 \\
Fuerza de prensión manual (kg) & $5,80(2,03)$ & $5,33(1,99)$ & $<0,001$ \\
\hline
\end{tabular}

DT: desviación típica. IMC: índice de masa corporal.

TABLA 4. Edad, variables antropométricas, estado nutricional, nivel de actividad física, tiempo de uso de pantallas y condición física según el nivel socioeconómico de los padres

\begin{tabular}{|c|c|c|c|c|c|}
\hline & $\begin{array}{c}\text { Bajo } \\
\text { Media (DT) }^{\mathrm{a}} \\
\mathrm{n}=102 \\
\end{array}$ & $\begin{array}{c}\text { Medio } \\
{\text { Media }(\mathrm{DT})^{\mathrm{b}}}^{\mathrm{n}=768} \\
\end{array}$ & $\begin{array}{c}\text { Alto } \\
{\text { Media }(\mathrm{DT})^{\mathrm{c}}}_{\mathrm{n}=\mathbf{2 9 3}}\end{array}$ & $p$-valor & $\begin{array}{l}\text { Prueba } \\
\text { post hoc }\end{array}$ \\
\hline Edad (meses) & $50,37(10,32)$ & $51,21(10,65)$ & $52,13(10,85)$ & 0,709 & \\
\hline $\mathrm{IMC}\left(\mathrm{kg} / \mathrm{m}^{2}\right)$ & $16,53(2,53)$ & $15,94(1,99)$ & $16,02(2,36)$ & 0,038 & $a>b^{*}$ \\
\hline Circunferencia de cadera $(\mathrm{cm})$ & $54,88(6,86)$ & $54,94(5,62)$ & $53,27(5,81)$ & 0,563 & \\
\hline Puntuación total Krece Plus (0-10) & $5,91(2,39)$ & $6,53(1,98)$ & $7,21(1,77)$ & $<0,001$ & $\mathrm{a}<\mathrm{b}^{*}, \mathrm{a}<\mathrm{c}^{* * *}, \mathrm{~b}<\mathrm{c}^{* * *}$ \\
\hline Actividad física semanal (horas) & $1,42(1,54)$ & $1,83(1,60)$ & $2,02(1,63)$ & 0,006 & $\mathrm{a}<\mathrm{c}^{* *}$ \\
\hline Tiempo de uso de pantallas diario (horas) & $3,29(6,15)$ & $3,70(8,01)$ & $1,85(0,93)$ & $<0,001$ & $\mathrm{~b}>\mathrm{c}^{* * *}$ \\
\hline Salto horizontal $(\mathrm{cm})$ & $70,32(28,29)$ & $72,13(26,44)$ & $61,50(22,50)$ & $<0,001$ & $a>c^{*}, b>c^{* * *}$ \\
\hline Velocidad, $20 \mathrm{~m}(\mathrm{~s})$ & $6,30(1,17)$ & $6,26(1,21)$ & $6,66(1,32)$ & $<0,001$ & $\mathrm{~b}<\mathrm{c}^{* * *}$ \\
\hline Resistencia (s) & $87,02(24,05)$ & $84,15(19,86)$ & $81,99(16,41)$ & 0,092 & \\
\hline Equilibrio (s) & $10,16(11,84)$ & $9,00(9,61)$ & $9,59(11,03)$ & 0,475 & \\
\hline Fuerza de prensión manual (kg) & $5,69(1,74)$ & $5,57(2,08)$ & $6,33(2,24)$ & 0,359 & \\
\hline
\end{tabular}

DT: desviación típica. IMC: índice de masa corporal. ${ }^{*} \mathrm{p}<0,05 ;{ }^{* *} \mathrm{p}<0,01 ;{ }^{* * *} \mathrm{p}<0,001$. 
El análisis de correlación de Pearson no reveló ninguna asociación significativa entre el tiempo de sedestación diario de los padres y el resto de las variables analizadas.

\section{DISCUSIÓN}

El hallazgo más relevante de este estudio indica que, en la población estudiada, el sexo de los niños y el nivel socioeconómico y de estudios de los padres afectaron a diferentes aspectos del estado ponderal, nutricional, AF y CF de niños preescolares.

En relación con el sexo, las niñas presentaron menor prevalencia de sobrepeso y obesidad que los niños; sin embargo, estos manifestaron mejor nivel de CF, y no afectó el sexo al estado nutricional, nivel de AF y uso de pantallas. Además, no se encontró asociación entre el IMC y el nivel de AF, tiempo de uso de pantallas, estado nutricional y, excepto con la fuerza de prensión manual, el resto de las pruebas de CF.

En relación con el estado nutricional y tomando como referencia el baremo de Serra et al., ${ }^{14}$ los niños preescolares de este estudio mostraron un nivel nutricional medio, lo que requería mejoras en la alimentación y la visita al pediatra en seis meses. Sin embargo, los niños con padres de nivel socioeconómico bajo y sin estudios presentaron un nivel nutricional muy bajo, lo que requería corregir urgentemente los hábitos alimentarios y la consulta con el pediatra.

En relación con los niveles de AF, los niños preescolares analizados en este estudio presentaron valores muy bajos de AF semanal, teniendo en cuenta las recomendaciones internacionales. ${ }^{16}$ Además, el tiempo de uso diario de pantallas fue superior al tiempo semanal de AF, lo que, unido a la alta prevalencia de sobrepeso y obesidad en relación con los datos de referencia de De Onis et al., ${ }^{17}$ expuso una población muy sensible a problemas de salud relacionados con el sobrepeso y el sedentarismo. En este sentido, Van Stralen et al., ${ }^{18}$ destacaron una asociación positiva entre el comportamiento sedentario, principalmente, el tiempo de uso de pantallas, y el IMC y la circunferencia de la cintura. Hinkley et al., ${ }^{19}$ indicaron que la mayoría de los niños pequeños no participaban en las cantidades adecuadas de AF y sí en cantidades excesivas de uso de pantallas. En relación con el sexo, otros autores, en consonancia con este estudio, no encontraron diferencias entre niños y niñas preescolares en los niveles de AF tanto si la evaluación se hacía por medios objetivos (acelerometría) como por informes parentales. ${ }^{20}$ Además, los niños con sobrepeso fueron significativamente menos activos, aunque no se observaron diferencias significativas en las niñas. ${ }^{21}$

Teniendo en cuenta la influencia de las características sociodemográficas de los padres en las diferentes variables, los niños con padres de nivel socioeconómico alto presentaron mejor estado nutricional, mayor tiempo de AF y menor uso de pantallas. Los niños de padres con estudios universitarios presentaron menor IMC, mejor estado nutricional y mayor salto horizontal. En

TABLA 5. Edad, variables antropométricas, estado nutricional, nivel de actividad física, tiempo de uso de pantallas y condición física según el nivel de estudios de los padres

\begin{tabular}{|c|c|c|c|c|c|c|}
\hline & $\begin{array}{c}\text { Sin estudios } \\
\text { Media (DT) } \\
n=26\end{array}$ & $\begin{array}{c}\text { Primarios } \\
{\text { Media }(D T)^{b}}^{\text {n }=289}\end{array}$ & $\begin{array}{c}\text { Secundarios } \\
\text { Media (DT) }^{\mathrm{c}} \\
\mathrm{n}=446\end{array}$ & $\begin{array}{l}\text { Universitarios } \\
\text { Media (DT) }^{\mathrm{d}} \\
\mathrm{n}=414\end{array}$ & p-valor & $\begin{array}{l}\text { Prueba } \\
\text { post hoc }\end{array}$ \\
\hline Edad (meses) & $48,00(8,72)$ & $51,47(10,65)$ & $51,54(11,07)$ & $50,53(10,29)$ & 0,327 & \\
\hline $\mathrm{IMC}\left(\mathrm{kg} / \mathrm{m}^{2}\right)$ & $16,88(3,21)$ & $16,28(2,28)$ & $15,95(2,17)$ & $15,86(1,90)$ & 0,017 & \\
\hline Circunferencia de cadera $(\mathrm{cm})$ & $54,30(5,79)$ & $54,67(5,48)$ & $54,84(6,34)$ & $55,07(5,22)$ & 0,879 & \\
\hline Puntuación total Krece Plus (0-10) & $5,92(2,93)$ & $6,55(1,99)$ & $6,66(1,98)$ & $6,85(1,89)$ & 0,042 & \\
\hline Actividad física semanal (horas) & $1,75(1,64)$ & $1,74(1,61)$ & $1,77(1,54)$ & $1,98(1,66)$ & 0,163 & \\
\hline $\begin{array}{l}\text { Tiempo de uso de } \\
\text { pantallas diario (horas) }\end{array}$ & $2,75(1,43)$ & $2,98(6,15)$ & $3,11(6,87)$ & $3,27(7,09)$ & 0,939 & \\
\hline Salto horizontal $(\mathrm{cm})$ & $64,41(29,41)$ & $65,28(25,60)$ & $69,75(25,37)$ & $71,97(26,68)$ & 0,009 & $\mathrm{~b}<\mathrm{d}^{* *}$ \\
\hline Velocidad, $20 \mathrm{~m}(\mathrm{~s})$ & $5,98(1,11)$ & $6,35(1,30)$ & $6,36(1,25)$ & $6,37(1,20)$ & 0,495 & \\
\hline Resistencia (s) & $90,88(23,96)$ & $83,04(18,20)$ & $83,69(19,75)$ & $84,19(19,73)$ & 0,281 & \\
\hline Equilibrio (s) & $11,69(10,49)$ & $10,07(11,36)$ & $9,36(10,30)$ & $8,72(9,68)$ & 0,255 & \\
\hline Fuerza de prensión manual (kg) & $5,90(1,55)$ & $5,44(2,07)$ & $5,62(1,93)$ & $5,59(2,13)$ & 0,707 & \\
\hline
\end{tabular}

DT: desviación típica. IMC: índice de masa corporal. ${ }^{* *} \mathrm{p}<0,01$. 
este sentido, Sotos et al., ${ }^{22}$ destacaron que tanto el nivel educacional como el socioeconómico influían en el estado nutricional infantil.

Además, en este estudio, el nivel de sedentarismo de los padres no se asoció con ninguna variable analizada. Sin embargo, Hinkley et al., ${ }^{23}$ señalaron que los niños con padres activos tendían a ser más activos. Del mismo modo, Hesketh et al., ${ }^{24}$ indicaron que los niveles de AF diaria de la madre se asociaban con todas las intensidades de AF de los niños preescolares.

Por tanto, la AF es uno de los factores que influyen en el sano desarrollo de los niños, pero la mayoría de los niños en edad preescolar tienden a ser inactivos. ${ }^{25} \mathrm{O}^{\prime} \mathrm{Dwyer}$ et al., ${ }^{26}$ sugirieron que la escuela representaba un entorno propicio para el sedentarismo. En España, sucede la misma circunstancia que señalan otros autore ${ }^{27}$ en relación con la AF en los colegios; así, aunque las escuelas pueden ofrecer oportunidades únicas para la AF estructurada, hay una tendencia a recortar las clases de Educación Física, debido a las presiones crecientes para mejorar los resultados académicos. Summerbell et al., ${ }^{28}$ recomendaron una serie de orientaciones para intervenir en el ámbito de la AF en niños preescolares, como fomentar el transporte activo para distancias cortas, visitar los lugares donde los niños pueden ser activos, desalentar la provisión de pantallas en el dormitorio, fomentar la participación no competitiva en la AF, mejorar todas las áreas de juego infantiles, dotar de ropa cómoda, proporcionar juegos durante las pausas de las clases, alentar a los niños a ser activos y disminuir el tiempo de sedentarismo total en el aula.

Una de las limitaciones de este estudio es su carácter transversal, lo que obliga a ser cautos en los resultados obtenidos y no permite realizar inferencias causales observadas. Otra es no haber considerado otros factores que pueden influir en las variables analizadas, por ejemplo, aspectos del entorno social y físico, tales como las instalaciones deportivas, la planificación urbana, los sistemas de transporte, parques y senderos, etc.

Sin embargo, una fortaleza de esta investigación es haber contado con una muestra amplia de sujetos y el registro de variables que escasamente se han abordado en estudios de esta naturaleza, como la CF.

Desde un punto de vista de aplicación práctica, intervenciones para mejorar el nivel de AF en niños preescolares, tanto en el colegio como fuera de este, son esenciales, todo ello acompañado con medidas de educación nutricional a los padres con menos recursos económicos y con escasa formación académica.

\section{CONCLUSIONES}

En conclusión, los niños preescolares de este estudio presentaron valores elevados de sobrepeso y obesidad y bajo nivel de AF, teniendo en cuenta las referencias internacionales. Las niñas mostraron una CF inferior a la de los niños. Por último, los niños cuyos padres tenían un nivel socioeconómico bajo y sin estudios mostraron un nivel nutricional precario.

\section{REFERENCIAS}

1. De Onis M, Onyango AW, Borghi E, Siyam A, et al. Development of a WHO growth reference for schoolaged children and adolescents. Bull World Health Organ 2007;85(9):660-7.

2. Bürgi F, Meyer U, Granacher U, Schindler C, et al. Relationship of physical activity with motor skills, aerobic fitness and body fat in preschool children: a cross-sectional and longitudinal study (Ballabeina). Int J Obes (Lond) 2011;35(7):937-44.

3. Te Velde SJ, Van Nassau F, Uijtdewilligen L, Van Stralen MM, et al. Energy balance-related behaviours associated with overweight and obesity in preschool children: a systematic review of prospective studies. Obes Rev 2012;13 Suppl 1(2):56-74.

4. DeBock F, Genser B, Raat H, Fischer JE, et al. A participatory physical activity intervention in preschools: a cluster randomized controlled trial. Am J Prev Med 2013;45(1):64-74.

5. Grzywacz JG, Suerken CK, Zapata Roblyer MI, Trejo G, et al. Physical activity of preschool-aged Latino children in farmworker families. Am J Health Behav 2014;38(5):717-25.

6. Tucker P. The physical activity levels of preschoolaged children: a systematic review. Early Child Res $Q$ 2008;23(4):547-58.

7. Ortega FB, RuizJR, Castillo MJ,Sjöström M. Physical fitness in childhood and adolescence: a powerful marker of health. Int J Obes (Lond) 2008;32(1):1-11.

8. Kondric M, Trajkovski B, Strbad M, Foretic N, et al. Anthropometric influence on physical fitness among preschool children: gender-specific linear and curvilinear regression models. Coll Antropol 2013;37(4):1245-52.

9. Ortega FB, Artero EG, Ruiz JR, España-Romero V, et al. Physical fitness levels among European adolescents: the HELENA study. Br J Sports Med 2011;45(1):20-9.

10. Travill AL. Correlation between growth and physical fitness of socially disadvantaged girls. South African Journal for Research in Sport, Physical Education and Recreation 2011;33(3):9069.

11. ReillyJJ. Descriptiveepidemiology and health consequences of childhood obesity. Best Pract Res Clin Endocrinol Metab 2005;19(3):327-41.

12. Singh AS, Mulder C, Twisk JW, Van Mechelen W, et al. Tracking of childhood overweightintoadulthood:asystematic review of the literature. Obes Rev 2008;9(5):474-88.

13. Latorre Román PA, Mora López D, Fernández Sánchez M, Salas Sánchez J, et al. Test-retest reliability of a field-based physical fitness assessment for children aged 3-6 years. Nutr Hosp 2015;32(4):1683-8.

14. Serra Majem L, Aranceta Bartrina J, Ribas Barba L, Sangil 
Monroy M, et al. Crecimiento y desarrollo: dimensión alimentaria y nutricional. Elcribado del riesgonutricionalen pediatría. Validación del test rápido Krece Plus y resultados en la población española. En: Serra Majem L, Aranceta Bartrina J, Rodríguez Santos F, eds. Crecimiento y desarrollo. Estudio enKid. Barcelona: Masson; 2003.Págs.45-51.

15. Craig CL, Marshall AL, Sjöström M, Bauman AE, et al. International physical activity questionnaire: 12-country reliability and validity. Med Sci Sports Exerc 2003;35(8): 1381-95.

16. Organización Mundial de la Salud. Recomendaciones mundiales sobre actividad física para la salud. Ginebra: Organización Mundial de la Salud; 2010.

17. De Onis M, Blössner M, Borghi E. Global prevalence and trends of overweight and obesity among preschool children. Am J Clin Nutr 2010;92(5):1257-64.

18. Van Stralen MM, Te Velde SJ, Van Nassau F, Brug J, et al. Weight status of European preschool children and associations with family demographics and energy balancerelated behaviours: a pooled analysis of six European studies. Obes Rev 2012;13(Suppl 1):29-41.

19. Hinkley T, Salmon J, Okely AD, Crawford D, et al. Preschoolers' physical activity, screen time, and compliance with recommendations. Med Sci Sports Exerc 2012;44(3): 458-65.

20. Taylor RW, Murdoch L, Carter P, Gerrard DF, et al. Longitudinal study of physical activity and inactivity in preschoolers: the FLAME study. Med Sci Sports Exerc 2009;41(1):96-102.
21. Trost SG, Sirard JR, Dowda M, Pfeiffer KA, et al. Physical activity in overweight and nonoverweight preschool children. Int J Obes Relat Metab Disord 2003;27(7):834-9.

22. Sotos-Prieto M, Santos-Beneit G, Pocock S, Redondo J, et al. Parental and self-reported dietary and physical activity habits in pre-school children and their socio-economic determinants. Public Health Nutr 2015;18(2):275-85.

23. Hinkley T,Crawford D, Salmon J,Okely AD, et al. Preschool children and physical activity. a review of correlates. Am J Prev Med 2008;34(5):435-41.

24. Hesketh KR, Goodfellow L, Ekelund U, McMinn AM, et al. Activity levels in mothers and their preschool children. Pediatrics 2014;133(4):e973-80.

25. Dolinsky DH, Brouwer RJN, Evenson KR, Siega-Riz AM, et al. Correlates of sedentary time and physical activity among preschool-aged children. Prev Chronic Dis 2011;8(6):A131.

26. O'Dwyer M, Fairclough SJ, Ridgers ND, Knowles ZR, et al. Patterns of objectively measured moderate-to-vigorous physical activity in preschool children. J Phys Act Health 2014;11(6):1233-8.

27. Singh A, Uijtdewilligen L, Twisk JW, van Mechelen W, et al. Physical activity and performance at school: a systematic review of the literature including a methodological quality assessment. Arch Pediatr Adolesc Med 2012;166(1):49-55.

28. Summerbell CD, Moore HJ, Vögele C, Kreichauf S, et al. Evidence-based recommendations for the development of obesity prevention programs targeted at preschoolchildren. Obes Rev 2012;13(Suppl 1):129-32. se encuentran disponibles en la versión electrónica de este número.

\section{JAMA 2016;315(18):1966-74}

Efecto del jugo de manzanas diluido y líquidos preferidos versus solución electrolítica de mantenimiento sobre el fracaso del tratamiento en niños con gastroenteritis moderada: ensayo clínico aleatorizado (Freedman SB, et al. Effect of dilute apple juice and preferred fluids vs electrolyte maintenance solution on treatment failure among children with mild gastroenteritis: a randomized clinical trial) Comentario: Dr. Alejandro Balestracci. Hospital General de Niños Pedro de Elizalde.

ARCH DIS CHILD 2016 May 25 [Epub ahead of print]

Consentimiento informado para investigación clínica pediátrica en Europa (Lepola P, et al. Informed consent for paediatric clinical trials in Europe)

Comentario: Dra. Fernanda M. Ledesma. Coordinadora Bioética Hospitalaria. Hospital de Pediatría J.P. Garrahan.

N ENGL J MED 2016;374(19):1822-30

Eventos graves relacionados con asma con fluticasona-salmeterol vs. fluticasona sola (Stempel DA, et al. Serious asthma events with fluticasone plus salmeterol versus fluticasone alone)

Comentario: Dr. Conrado Llapur. Hospital del Niño Jesús. Facultad de Medicina, Universidad Nacional de Tucumán.

ARCH DIS CHILD 2016 June 23 [Epub ahead of print]

Comparación del tiempo de llenado capilar central y periférico en niños febriles que concurren a un servicio de emergencia pediátrico y su utilidad en la identificación de niños con infección bacteriana grave (de Vos-Kerkhof $E$ et al. Comparision of peripheral and central capillary refill time in febrile children presenting to a paediatric emergency department and its utility in identifying children with serious bacterial infection.

Comentario: Dr. Luis Eduardo Urrutia. Hospital de Pediatría "Prof. Dr. Juan P. Garrahan" 